\title{
Reopening of L-type calcium channels in human ventricular myocytes during applied epicardial action potentials
}

\author{
L. Fülöp,' T. Bányász,' J. Magyar,' ' N. Szentandrássy,' A. Varró ${ }^{2}$ and P. P. Nánási' \\ Department of Physiology, University Medical School of Debrecen, Debrecen, Hungary \\ 2 Department of Pharmacology and Pharmacotherapy, University of Szeged, Szeged, Hungary
}

Received 19 April 2002, accepted 24 September 2003 Correspondence: P. P. Nánási, Department of Physiology, University of Debrecen, Debrecen, H-40 12, P.O. Box 22, Hungary.

\begin{abstract}
Aims: Present study was performed to compare the dynamics of human L-type calcium current $\left(I_{\mathrm{Ca}, \mathrm{L}}\right)$ flowing during rectangular voltage pulses, voltage ramps, and action potentials (APs) recorded from epicardiac and endocardiac canine ventricular cells.

Methods: $I_{\mathrm{Ca}, \mathrm{L}}$ was recorded in single myocytes isolated from undiseased human hearts using the whole cell voltage clamp technique.

Results: The decay of $I_{\mathrm{Ca}, \mathrm{L}}$ was monotonic when using rectangular pulses or endocardial APs as voltage commands, whereas the current became doublepeaked (displaying a second rise and fall) during epicardial (EPI) APs or voltage ramps used to mimic EPI APs. These $I_{\mathrm{Ca}, \mathrm{L}}$ profiles were associated with single-hooked and double-hooked phase-plane trajectories, respectively. No sustained current was observed during the AP commands. Kinetics of deactivation and recovery from inactivation of human $I_{\mathrm{Ca}, \mathrm{L}}$ were determined using twin-pulse voltage protocols and voltage ramps, and the results were similar to those obtained previously in canine cells under identical experimental conditions.

Conclusions: $I_{\mathrm{Ca}, \mathrm{L}}$ can inactivate partially before and deactivate during the phase-1 repolarization of the epicardiac AP, and reopening of these channels seems to be associated with formation of the dome.

Keywords action potential, calcium current, human myocytes, regional differences, voltage clamp.
\end{abstract}

Characteristic differences in action potential (AP) configuration have been explored in cardiac myocytes originating from various layers of the mammalian ventricular wall (Litovsky \& Antzelevitch 1988, Stankovicova et al. 2000). These differences are primarily because of the asymmetrical distribution of various potassium currents, primarily $I_{\text {to, (Furukawa }}$ et al. 1992, Liu et al. 1993, Wettwer et al. 1994, Liu \& Antzelevitch 1995, Brahmajothi et al. 1999, Volk et al. 1999). In canine ventricular cells, where the transmural heterogeneity in AP morphology is prominent, endocardial (ENDO) cells display a pronounced plateau, while epicardial (EPI) cells produce a spike-and-dome AP configuration (Litovsky \& Antzelevitch 1990,
Krishnan \& Antzelevitch 1991). It has recently been published that $I_{\mathrm{Ca}, \mathrm{L}}$ is double-peaked, displaying a second rise and fall, during canine APs of EPI - but not ENDO - origin when studied under AP voltage clamp conditions, in spite of the fact that no differences in current density and kinetics were detected in $I_{\mathrm{Ca}, \mathrm{L}}$ between EPI and ENDO cells when compared with conventional voltage clamp (Bányász et al. 2003). Therefore, Bányász et al. (2003) concluded that the EPI-ENDO differences observed in $I_{\mathrm{Ca}, \mathrm{L}}$ profiles were consequences of the different AP configurations. It was also shown in the same study that calcium channels can reopen during the crest of the dome of the AP in EPI canine myocytes. 
As the transmural differences in both $I_{\text {to }}$ density (Wettwer et al. 1994) and AP configuration (Nabauer et al. 1996) in human ventricular myocytes were shown to be similar to those observed previously in canine cells, it was reasonable to predict that the EPI-ENDO differences in the $I_{\mathrm{Ca}, \mathrm{L}}$ profile might also be similar in the two species. If this is the case, reopening of calcium channels can also be demonstrated in human myocytes. To test this hypothesis we applied the combination of the conventional whole-cell voltage clamp and AP voltage clamp techniques in ventricular cells isolated from five undiseased human hearts. This latter method offers a valuable approach to study the dynamics of a specific ion current as it is actually displayed during the cardiac AP (Doerr et al. 1990, Arreola et al. 1991, Ibarra et al. 1991), whereas the kinetic properties of $I_{\mathrm{Ca}, \mathrm{L}}$ were determined using rectangular voltage pulses. It was found that (1) calcium channels in human ventricular myocytes can reopen during an EPI AP, (2) the kinetic properties of $I_{\mathrm{Ca}, \mathrm{L}}$ in human myocytes are very similar to those obtained earlier in canine cells by Bányász et al. (2003), and (3) the EPI-ENDO differences we observed earlier in the $I_{\mathrm{Ca}, \mathrm{L}}$ profile in dog are likely to be present in human ventricular myocardium as well.

\section{Methods}

\section{Isolation of single human ventricular myocytes}

Human ventricular cells were prepared from five donor hearts. The hearts were obtained from general organ donor patients whose semilunar valves were used for transplantation. Before explantation, the patients did not receive any medication other than dobutamine, furosemide and plasma expanders. The investigation conformed to the principles outlined in the Declaration of Helsinki, and the experimental protocol was approved by the local ethical committee.

Single myocytes were obtained by enzymatic dispersion using the segment perfusion technique (Magyar et al. 2000). Briefly, a wedge-shaped section of the ventricular wall supplied by the left anterior descending coronary artery was dissected, cannulated and perfused with oxygenated Tyrode solution containing: $\mathrm{NaCl} 144$, $\mathrm{KCl}$ 5.6, $\mathrm{CaCl}_{2}$ 2.5, $\mathrm{MgCl}_{2}$ 1.2, N-2-hydroxyethylpiperazine-N-2-ethane-sulfonic acid (HEPES) 5, and dextrose $11 \mathrm{mmol} \mathrm{L}^{-1}$ at $\mathrm{pH}=7.4$. Perfusion was maintained until the removal of blood from the coronary system and then switched to a nominally calciumfree Joklik solution (Minimum Essential Medium Eagle, Joklik Modification; Sigma, St Louis, MO, USA) for $5 \mathrm{~min}$. This was followed by $30 \mathrm{~min}$ perfusion with re-circulated Joklik solution supplemented with
$1 \mathrm{mg} \mathrm{mL}^{-1}$ collagenase (Type II; Worthington, Chemical Co., Lakewood, NJ, USA) and $0.2 \%$ bovine serum albumine (Fraction V; Sigma) containing $50 \mu \mathrm{mol} \mathrm{L}^{-1}$ calcium. Then the left ventricular wall were cut into small pieces and the cell suspension obtained at the end of the procedure was washed with Joklik solution and the calcium concentration was gradually increased to $2.5 \mathrm{mmol} \mathrm{L}^{-1}$. The cells were stored in Minimum Essential Medium Eagle supplemented with taurine 20, pyruvic acid 2, ribose 5, allopurinol 0.1, $\mathrm{NaHCO}_{3}$ 26 and $\mathrm{NaH}_{2} \mathrm{PO}_{4} 1.5 \mathrm{mmol} \mathrm{L}^{-1}$ until use.

\section{Electrophysiology}

All electrical recordings were performed using the whole-cell configuration of the patch clamp technique (Hamill et al. 1981). Myocytes were transferred to a Plexiglass chamber of $0.5 \mathrm{~mL}$ volume, which was mounted on the stage of an inverted microscope and superfused with Tyrode solution at $37^{\circ} \mathrm{C}$. The flow rate was set to $10 \mathrm{~mL} \mathrm{~min}{ }^{-1}$. All cells were rod shaped and showed clear striation. Electrodes were prepared from borosilicate glass, having resistance of 1.5-2.5 M $\Omega$ when filled with pipette solution (containing: $\mathrm{K}$-aspartate $100, \mathrm{KCl} 45, \mathrm{MgCl}_{2} 1$, ethyleneglycoltetraacetic acid (EGTA) 10, K-ATP 3, and HEPES $5 \mathrm{mmol} \mathrm{L}^{-1}$, for AP voltage clamp, or $\mathrm{KCl} 110, \mathrm{KOH} 40$, TEACl 20, HEPES 10, K-ATP 3, EGTA 10, and GTP $0.25 \mathrm{mmol} \mathrm{L}^{-1}$ for conventional voltage clamp experiments, at $\mathrm{pH}=7.4$ ). Application of the former pipette solution allowed recording of APs, whereas the TEACl content of the latter effectively blocked potassium currents. The liquid junction potential was $<3 \mathrm{mV}$ in the conventional voltage clamp experiments, this difference was ignored when presenting the results. For the AP voltage clamp measurements, the junction potential was calculated to be $12.6 \mathrm{mV}$, therefore, these results (presented in Fig. 1) are corrected for the liquid junction potential. However, it is important to note, that the AP waveform, delivered to the cell as a voltage command, was not corrected for the junction potential, as the same junction potential was experienced by the membrane when either recording or delivering the AP waveform.

The patch pipette was positioned to the middle of the cell in order to minimize inhomogeneity of the membrane potential, and consequently, to better approximate space clamp conditions. Careful suction was applied to help gigaseal formation and the subsequent disruption of the membrane patch. Axoclamp 2B amplifier (Axon Instruments, Foster City, CA, USA) was used in current clamp or continuous single electrode voltage clamp mode. Data were digitally sampled at $50 \mathrm{kHz}$ and filtered at $10 \mathrm{kHz}$ using the lowpass Bessel filter of the amplifier. Digidata 1200 AD/DA 

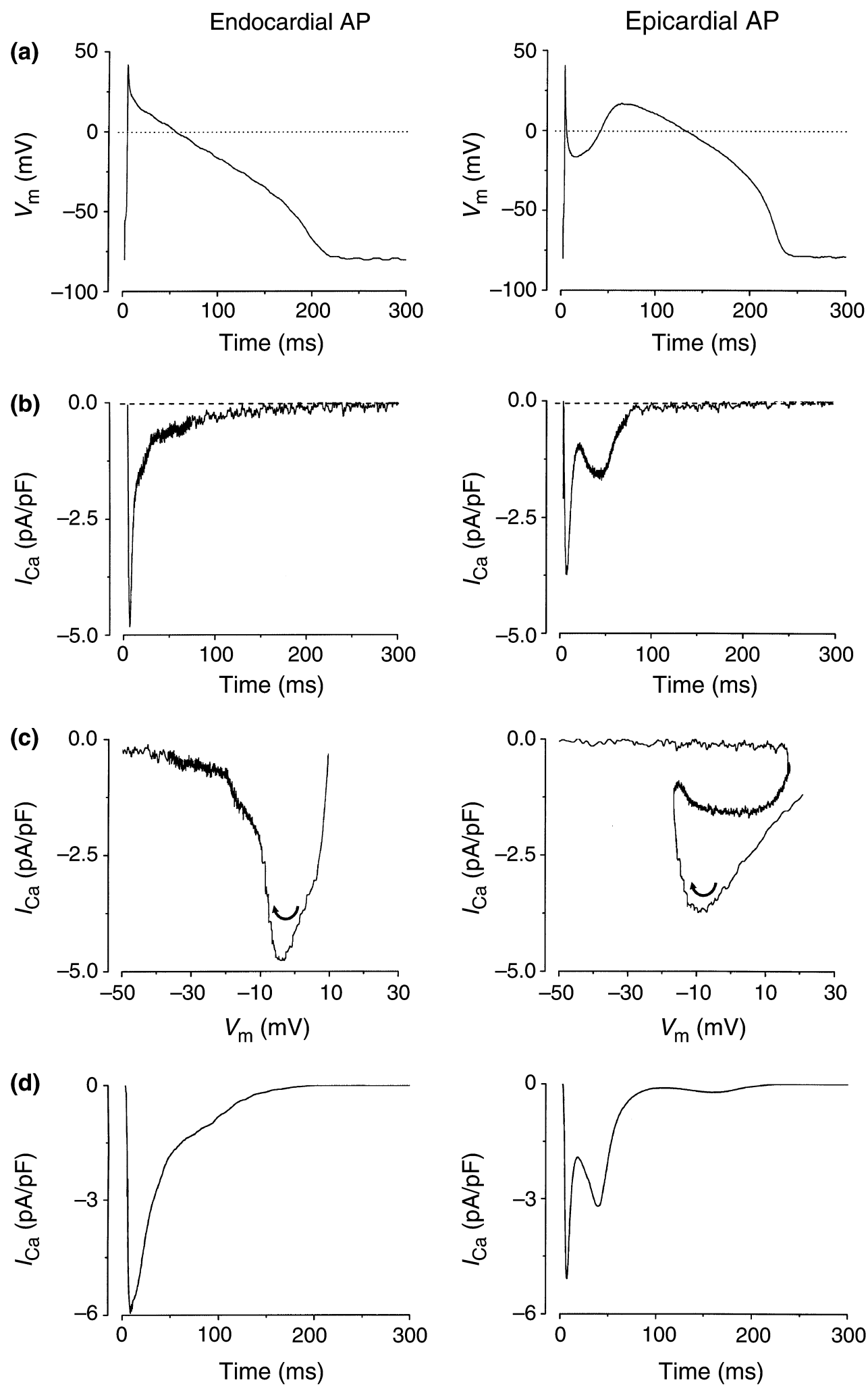

Figure I Endocardial (left panels) and epicardial (right panels) canine action potentials (APs), used as voltage commands (a), and the corresponding $I_{\mathrm{Ca}, \mathrm{L}}$ profiles (b) and current-voltage relationships (c) recorded under AP voltage clamp conditions in a human ventricular cell. $I_{\mathrm{Ca}, \mathrm{L}}$ was measured as nisoldipine-sensitive current. Current-voltage relationship for $I_{\mathrm{Ca}, \mathrm{L}}$ was obtained by plotting the nisoldipine-sensitive current against isochronal membrane potential values derived from the AP, where arrows indicate the sequence of events. (d) Computer simulation of $I_{\mathrm{Ca}, \mathrm{L}}$ obtained by feeding the actually used command APs into the Luo-Rudy model. 
converter operated under pClamp 6.0 software (Axon Instruments) was used to collect data and to deliver voltage clamp protocols. Ionic currents were normalized to cell capacitance $(162.8 \pm 16 \mathrm{pF})$, determined in each cell using hyperpolarizing pulses from -10 to $-20 \mathrm{mV}$ for $40 \mathrm{~ms}$. The series resistance was typically 4-8 $\mathrm{M} \Omega$ before compensation (usually 50-80\%).

In conventional voltage clamp experiments $I_{\mathrm{Ca}, \mathrm{L}}$ was measured during $100 \mathrm{~ms}$ long depolarizations to $+10 \mathrm{mV}$ arising from the holding potential of $-40 \mathrm{mV}$. Peak current density was defined as a difference between the peak value of $I_{\mathrm{Ca}, \mathrm{L}}$ and its pedestal measured at the end of the pulse. The applied twin pulse and voltage ramp protocols will be described where appropriate in the Results section.

The APs, recorded previously from canine ventricular myocytes of EPI and ENDO origin, were applied as command signals in the AP voltage clamp studies assuming that a canine AP represents good model for a human AP. We used canine APs because of the following reasons: (1) we had no exact information on the actual origin of our human cells within the ventricular wall, (2) the dispersed human cells were slightly depolarized (to around $-70 \mathrm{mV}$ ) and (3) AP configuration is very similar in human and canine ventricular myocytes (Liu et al. 1993, Nabauer et al. 1996). $I_{\mathrm{Ca}, \mathrm{L}}$ was identified as a nisoldipine-sensitive current, appearing with an inverse polarity after application of $1 \mu \mathrm{mol} \mathrm{L}{ }^{-1}$ nisoldipine (Zaza et al. 1998). Subtraction of current traces obtained in the absence and presence of nisoldipine yielded traces of $I_{\mathrm{Ca}, \mathrm{L}}$ with the normal, inwardly directed polarity. Nisoldipine was obtained from Bayer (Leverkusen, Germany), other drugs from Sigma Chemicals.

\section{Results}

\section{$I_{C a, L}$ profile in human ventricular myocytes under AP voltage clamp conditions}

The profile of $I_{\mathrm{Ca}, \mathrm{L}}$ (defined as a nisoldipine-sensitive current) during the AP was determined under AP voltage clamp conditions (Fig. 1). The current displayed a sharp upstroke and rapid decay when applying either ENDO or EPI AP as a command signal. Following its decay $I_{\mathrm{Ca}, \mathrm{L}}$ increased again during EPI but not ENDO APs (Fig. 1b). This second rise of $I_{\mathrm{Ca}, \mathrm{L}}$ vas evident after the deepest point of the incisura of the AP and peaked before the crest of the dome. The amplitude of the second current peak was smaller than that of the first one. Current-voltage relationship of $I_{\mathrm{Ca}, \mathrm{L}}$ was constructed by plotting the current as a function of the membrane potential. As shown by the phase-plane trajectories (displayed in Fig. 1c) the current built up within a narrow range of membrane potential, then decreased during both types of APs. This decline was monotonic and complete in case of an ENDO AP, whereas the second rise of $I_{\mathrm{Ca}, \mathrm{L}}$ formed an additional loop on the phase-plane trajectory around $0 \mathrm{mV}$ when EPI APs were used as command signals. Finally, $I_{\mathrm{Ca}, \mathrm{L}}$ fell below $50 \mathrm{pA}$ at potentials negative to $-10 \mathrm{mV}$, indicating that only small sustained current remained active during the plateau. Similar results were obtained in each of the three human myocytes exposed to canine APs of EPI and ENDO origin. The ENDO and EPI APs, being used as command signals in the AP clamp experiments, were also fed into the Luo-Rudy model (completed with Kass-Sanguinetti inactivation kinetics) in order to simulate $I_{\mathrm{Ca}, \mathrm{L}}$. These traces of $I_{\mathrm{Ca}, \mathrm{L}}$ obtained by computer simulations (shown in Fig. 1d) strongly resemble the experimentally recorded ones (Fig. 1b). The results suggest that the characteristic single-peaked or double-peaked appearance of the $I_{\mathrm{Ca}, \mathrm{L}}$ profile, observed during an AP of ENDO or EPI origin, respectively, is likely to be determined by the configuration of the AP experienced.

\section{Properties of $I_{C a, L}$ in human ventricular cells under conventional voltage clamp}

To explain the mechanism of the second activation of $I_{\mathrm{Ca}, \mathrm{L}}$, observed in human ventricular cells when applying EPI APs as command pulses under AP voltage clamp conditions, we assumed that part of $I_{\mathrm{Ca}, \mathrm{L}}$ deactivates during phase-1 repolarization and this population of deactivated channels may reopen during the crest of the dome. Also, another fraction of $I_{\mathrm{Ca}, \mathrm{L}}$ which has already been inactivated before the early repolarization may recover from inactivation during the incisura. To test this hypothesis the voltage-dependence of deactivation and recovery from inactivation of $I_{\mathrm{Ca}, \mathrm{L}}$ was studied using rectangular voltage pulses as command signals. In these experiments $I_{\text {to }}$ was blocked by $3 \mathrm{mmol} \mathrm{L}^{-1}$ 4-aminopyridine added to the bathing solution.

In one series of experiments (Fig. $2 \mathrm{a}-\mathrm{c}$ ) two depolarizing pulses $\left(\mathrm{P}_{1}\right.$ and $\mathrm{P}_{2}$, having durations of 25 or $100 \mathrm{~ms}$, and $100 \mathrm{~ms}$, respectively) were delivered from -40 to $+10 \mathrm{mV}$ at a cycle length of $5 \mathrm{~s}$. These depolarizing pulses were separated by repolarizations to potentials ranging from +10 to $-40 \mathrm{mV}$ for either 25 or $100 \mathrm{~ms}$. The $25 \mathrm{~ms}$ period of repolarization mimicked the incisura of an EPI AP, and was applied to demonstrate that the availability of $I_{\mathrm{Ca}, \mathrm{L}}$ will increase with the degree of repolarization during the incisura. The underlying current records show $I_{\mathrm{Ca}, \mathrm{L}}$ traces elicited by the first pulse and then interrupted by the repolarizing steps (Fig. 2a). Although the current increased transiently at the beginning of the repolarizing step due to the increased driving force for $I_{\mathrm{Ca}, \mathrm{L}}$, it fell to values close to zero within a few milliseconds, due to 
(a)

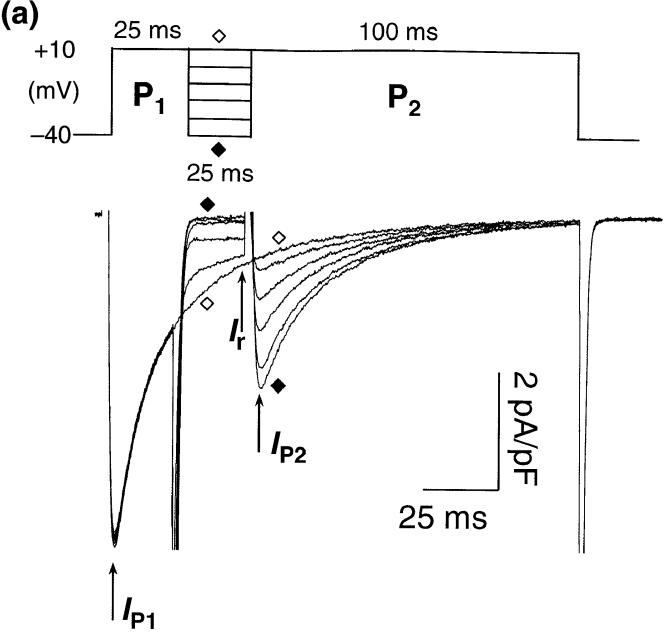

(d)

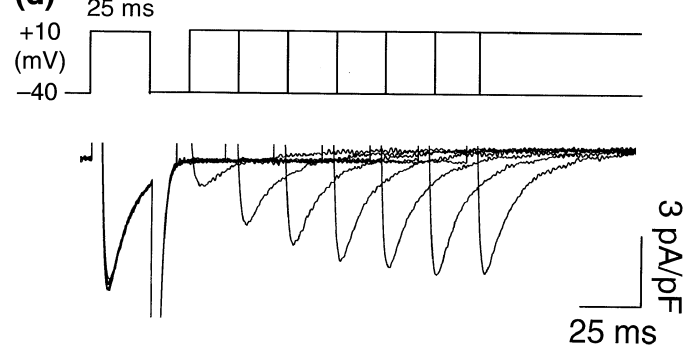

(b)

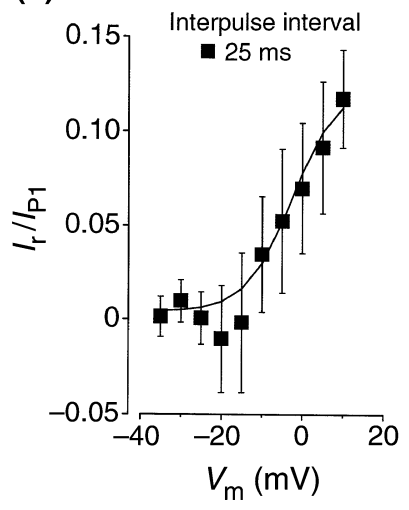

(c)

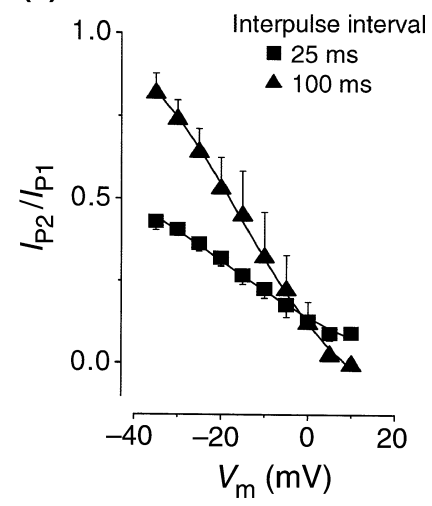

(e)

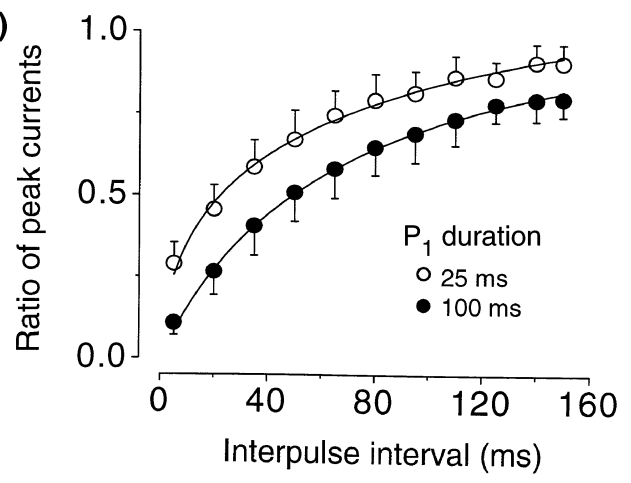

Figure 2 Deactivation and recovery kinetics of $I_{\mathrm{Ca}, \mathrm{L}}$ in human ventricular myocytes $(n=4)$. Two rectangular depolarizing voltage pulses $\left(\mathrm{P}_{1}\right.$ and $\left.\mathrm{P}_{2}\right)$ were delivered to $+10 \mathrm{mV}$ from the holding potential of $-40 \mathrm{mV}$ (a). These pulses were separated by interpulse intervals, lasting for 25 or $100 \mathrm{~ms}$, and clamped to potentials ranging between -40 and $+10 \mathrm{mV}$. The current peaks measured during $\mathrm{P}_{1}$ and $\mathrm{P}_{2}$ are $I_{\mathrm{P} 1}$ and $I_{\mathrm{P} 2}$, respectively, and the current measured at the end of the interpulse interval was termed as $I_{\mathrm{r}}$. Ratios of $I_{\mathrm{r}} / I_{\mathrm{P} 1}(\mathrm{~b})$ and $I_{\mathrm{P} 2} / I_{\mathrm{P} 1}$ (c) were plotted as a function of the interpulse potential to describe the voltage-dependence of deactivation and recovery from inactivation, respectively. Solid lines were obtained by fitting data to the Boltzmann function. (d, e) Time course of recovery from inactivation of $I_{\mathrm{Ca}, \mathrm{L}}$, measured using twin-pulse protocol, where the duration of the first pulse $\left(\mathrm{P}_{1}\right)$ was either 25 or $100 \mathrm{~ms}$. The interpulse interval was gradually increased up to $150 \mathrm{~ms}$. The peak current measured during the second pulse was normalized to that measured during the first one and these current ratios were plotted against the interpulse interval. Solid lines represent fits to single exponentials. Symbols and bars are mean \pm SEM values.

voltage-dependent deactivation. The voltage-dependence of this deactivation can be best assessed by plotting the $I_{\mathrm{r}} / I_{\mathrm{P} 1}$ ratios, shown in Fig. $2 \mathrm{~b}$, as a function of the interpulse potential (using the shorter, $25 \mathrm{~ms}$ long $\mathrm{P}_{1}$ duration and $25 \mathrm{~ms}$ interpulse interval), where $I_{\mathrm{r}}$ is the current measured at the end of the interpulse interval and $I_{\mathrm{P} 1}$ is the peak current recorded during the first pulse. Fitting results to the Boltzmann function yielded a midpoint potential of $-12.7 \pm 0.5 \mathrm{mV}$ and a slope factor of $5.7 \pm 2.8 \mathrm{mV}(n=4)$. These results indicate that deactivation of $I_{\mathrm{Ca}, \mathrm{L}}$ develops at the membrane potential range covered by the incisura.

It was hypothesized that both pools of calcium channels (i.e. those closed via deactivation during the interpulse interval and those which closed via inactivation during the first depolarization) may contribute to the calcium channel pool available for activation during the second depolarization. Figure $2 c$ illustrates the relation between the interpulse potential and the peak amplitude of $I_{\mathrm{Ca}, \mathrm{L}}$ measured during the second pulse $\left(I_{\mathrm{P} 2}\right)$ and normalized to $I_{\mathrm{P} 1}$. With increasing the hyperpolarizing voltage prior to the second depolarization the peak amplitude of $I_{\mathrm{Ca}, \mathrm{L}}$ increased in a voltage-dependent manner. Lengthening the interpulse interval from 25 to $100 \mathrm{~ms}$ also increased the amplitude of the second peak of $I_{\mathrm{Ca}, \mathrm{L}}$ indicating an increasing contribution of previously inactivated and reopening $\mathrm{Ca}^{2+}$ channels to the second current peak. The voltage-dependence of recovery from inactivation was assessed by plotting the $I_{\mathrm{P} 2} / I_{\mathrm{P} 1}$ ratios as a function of the interpulse potential using the longer $(100 \mathrm{~ms})$ interpulse interval. A midpoint potential of $-15.3 \pm 2.0 \mathrm{mV}$ and a slope factor of $13.2 \pm 2.6 \mathrm{mV}(n=4)$ was obtained by fitting the results to the Boltzmann function. The time course of recovery from inactivation of $I_{\mathrm{Ca}, \mathrm{L}}$ was determined using the twin-pulse protocol shown in Figure 2d. The 
interpulse interval, following the first depolarization, (having $\mathrm{P}_{1}$ duration of either 25 or $100 \mathrm{~ms}$ ), was continuously varied from 5 to $150 \mathrm{~ms}$. The shorter (25 ms) prepulse was applied to approximate conditions occurring during an AP, while the longer $(100 \mathrm{~ms})$ prepulse was used to fully inactivate the current allowing the determination of its recovery time constant. The ratio of peak currents $\left(I_{\mathrm{P} 2} / I_{\mathrm{P} 1}\right)$ was plotted against the interpulse interval and the time constant for recovery was estimated by fitting data with a single exponential (shown in Fig. 2e) yielding a $61.9 \pm 2.1 \mathrm{~ms}$ time constant for recovery of $I_{\mathrm{Ca}, \mathrm{L}}$ from inactivation $(n=4)$. It is important to note that the recovery curve obtained using $25 \mathrm{~ms} \mathrm{P}_{1}$ duration started from a non-zero value indicating that a fraction of channels (which failed to inactivate within the $25 \mathrm{~ms}$ duration of the prepulse, consequently, closed via deactivation after the prepulse) was available for activation immediately after the prepulse. Figure $2 \mathrm{e}$ also suggests that the fraction of $I_{\mathrm{Ca}, \mathrm{L}}$ available for activation during the crest of the dome of the AP will increase with increasing the duration of the incisura.

Further parameters to characterize human $I_{\mathrm{Ca}, \mathrm{L}}$ were also determined under conventional voltage clamp conditions (not shown). The current-voltage relationship of $I_{\mathrm{Ca}, \mathrm{L}}$ was studied using a series of $200 \mathrm{~ms}$ long depolarizations to test potentials increasing in $5 \mathrm{mV}$ steps from -40 to $+60 \mathrm{mV}$. The current first appeared at $-20 \mathrm{mV}$, its maximum was reached at $+10 \mathrm{mV}$, and the reversal potential was found at $+50 \mathrm{mV}$. The density of peak $I_{\mathrm{Ca}, \mathrm{L}}$ in human myocytes was $-5.5 \pm 0.4 \mathrm{pA} \mathrm{pF}^{-1}$ at $+10 \mathrm{mV}(n=8)$. The voltage-dependence of steadystate inactivation was assessed using test depolarizations to $+10 \mathrm{mV}$ preceded by a set of prepulses clamped to various voltages between -55 and $+20 \mathrm{mV}$ for $500 \mathrm{~ms}$. Peak currents measured after these prepulses were normalized to the peak current measured after the $-55 \mathrm{mV}$ prepulse and plotted against the respective prepulse potential. Fitting the data to the Boltzmann function yielded $-20.9 \pm 0.4 \mathrm{mV}$ for midpoint potential of steady-state inactivation and $3.8 \pm 0.3 \mathrm{mV}$ for its slope factor $(n=7)$. The time constant of current decay (inactivation) was fitted as a sum of two exponential components. Time constants of $12.2 \pm$ 0.5 and $82.8 \pm 4.6 \mathrm{~ms}$ were obtained for the faster and slower components, having relative contributions of $87 \pm 3$ and $13 \pm 1.2 \%$, respectively, at $+10 \mathrm{mV}$ $(n=4)$.

These results obtained for peak current density, voltage- and time-dependence of inactivation and recovery, and voltage-dependence of deactivation well corresponded to those measured earlier by Bányász et al. (2003) in canine myocytes under identical experimental conditions.

\section{Analysis of human $I_{C a, L}$ using ramp protocol}

To mimic the actual spike-and-dome configuration of an EPI AP, a ramp protocol, shown in Figure 3a, was used. After clamping the membrane potential to $+10 \mathrm{mV}$ for $2.5 \mathrm{~ms}$ (from the holding potential of $-40 \mathrm{mV}$ ), a series of repolarizing voltage ramps were applied to potentials ranging from +10 to $-40 \mathrm{mV}$ for $5 \mathrm{~ms}$. These ramps were followed by longer $(20 \mathrm{~ms})$ ramps to the opposite direction, and after this the membrane potential was set again to $+10 \mathrm{mV}$. An additional advantage of this protocol was that it allowed a better analysis of deactivation kinetics of $I_{\mathrm{Ca}, \mathrm{L}}$ as the current was less distorted by inactivation induced by the initial depolarizing pulse. As shown in Figure $3 \mathrm{a}, I_{\mathrm{Ca}, \mathrm{L}}$ was activated by the initial short depolarization, and the current rapidly decayed during the rapid repolarizing ramps because of deactivation of calcium channels. The second, slower, depolarizing ramp (which was applied to mimic the rising phase of the dome) caused reopening of the channels.

Voltage-dependence of deactivation was assessed by normalizing the value of the minimum current, $I_{\mathrm{r}}$ (or the current measured at the time of the minimum current of the most negative voltage ramp) to the corresponding first $I_{\mathrm{Ca}}$ peak $\left(I_{\mathrm{P} 1}\right)$ and these $I_{\mathrm{r}} / I_{\mathrm{P} 1}$ ratios were plotted as a function of the endpoint potential of the repolarizing ramp (Fig. 3b). Fitting the data to the Boltzmann function yielded $-9.6 \pm 0.3 \mathrm{mV}$ for the midpoint potential and $9.5 \pm 0.3 \mathrm{mV}$ for the slope factor $(n=4)$. The estimated minimum and maximum amplitudes for the $I_{\mathrm{r}} / I_{\mathrm{P} 1}$ ratios were $0.042 \pm 0.01$ and $0.84 \pm 0.01$, respectively. The relation between the endpoint potential of the repolarizing ramp and the amplitude of the second $I_{\mathrm{Ca}, \mathrm{L}}$ peak (normalized to that of the first one as $I_{\mathrm{P} 2} / I_{\mathrm{P} 1}$ ) is shown in Figure 3c. The results indicate that increasing the depth of the hyperpolarizing voltage ramp (corresponding to a deeper incisura on the EPI AP) increases the amplitude of the second $I_{\mathrm{Ca}, \mathrm{L}}$ peak in a voltage-dependent manner.

Finally, we modelled the time course of $I_{\mathrm{Ca}, \mathrm{L}}$ flowing during our ramp protocol tested experimentally above. For computation of the $I_{\mathrm{Ca}, \mathrm{L}}$ profile we used kinetic parameters published by Luo \& Rudy (1994) completed with inactivation kinetics of Kass \& Sanguinetti (1984). The results of these simulations, shown in Figure 3d-f, were similar to those obtained experimentally: the midpoint potential and slope factor calculated for voltage-dependent deactivation of $I_{\mathrm{Ca}, \mathrm{L}}$ were -17.7 and $6.4 \mathrm{mV}$, respectively.

\section{Discussion}

The major result of this study was to show that calcium channels inactivate before and deactivate during the 
(a)

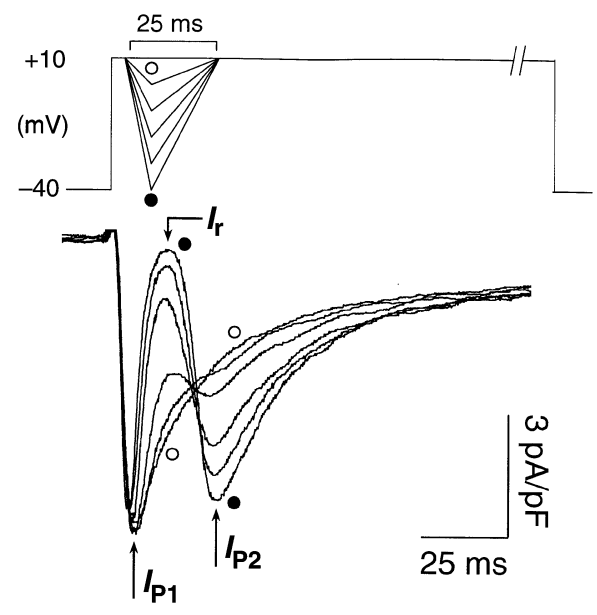

(b)

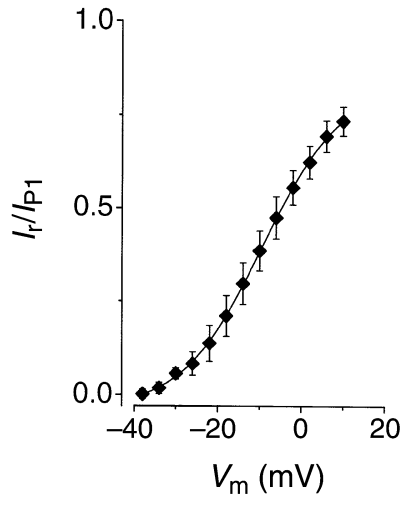

(d)

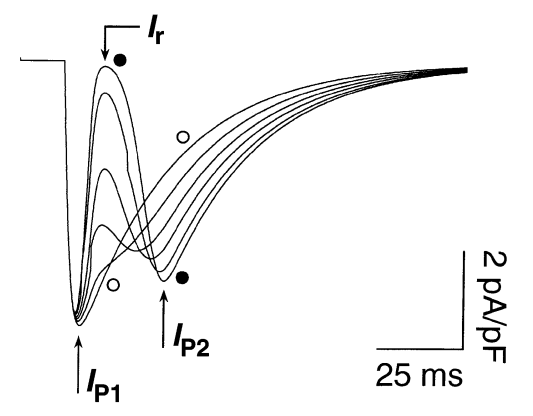

(e)

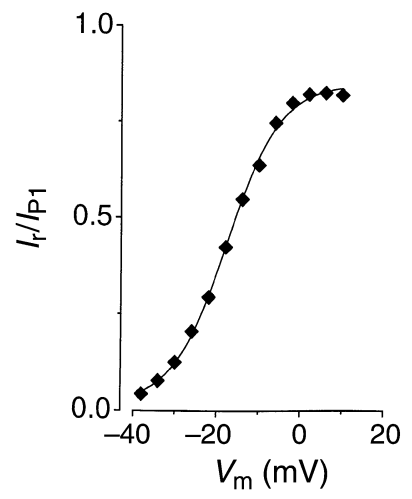

(c)
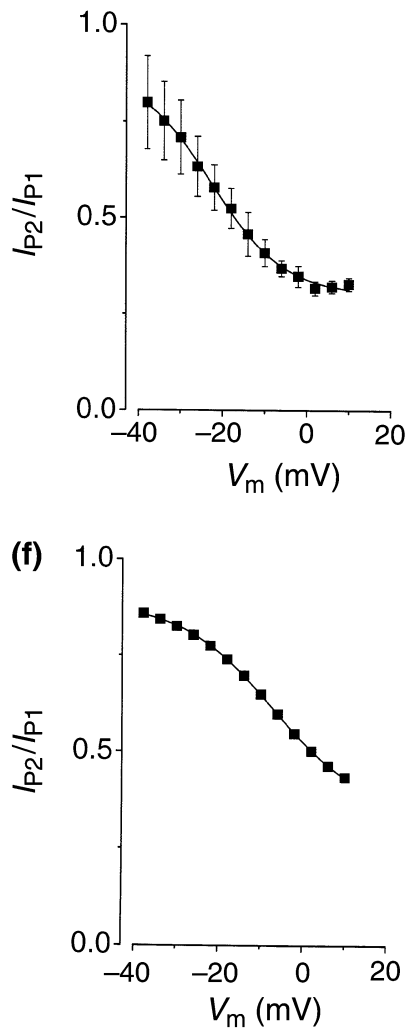

Figure 3 Evidence for voltage-dependent deactivation and reopening of L-type calcium channels in human ventricular myocytes. $(\mathrm{a}-\mathrm{c})$ Show results obtained experimentally in four myocytes, while (d, e) represent computer simulations using the Luo-Rudy model completed with Kass-Sanguinetti inactivation kinetics. In both cases a ramp protocol was applied to mimic the action potential waveform of an epicardial cell. After clamping the membrane potential to $+10 \mathrm{mV}$ for $2.5 \mathrm{~ms}$, a series of $5 \mathrm{~ms}$ long repolarizing voltage ramps were followed by $20 \mathrm{~ms}$ ramps to the opposite direction, and finally the membrane potential returned to $+10 \mathrm{mV}$. The endpoint potential of the repolarizing ramps [displayed in the abscissa of (b), (c), (e) and (f)] was varied between +10 and $-40 \mathrm{mV} . I_{\mathrm{P} 1}$ and $I_{\mathrm{P} 2}$ are amplitudes of the first and second $I_{\mathrm{Ca}, \mathrm{L}}$ peak, respectively, $I_{\mathrm{r}}$ values are minimum currents measured on the effect of ramp repolarization.

incisura of an EPI AP in human ventricular myocytes, and these channels do reopen during the crest of the dome. Accordingly, it has also been demonstrated that the profile of $I_{\mathrm{Ca}, \mathrm{L}}$ is strictly determined by the AP configuration: single-peaked and double-peaked $I_{\mathrm{Ca}, \mathrm{L}}$ profiles were obtained on the same human cells when canine APs of ENDO or EPI origin were applied as command pulses under AP voltage clamp conditions. These findings are fully congruent with recent results obtained in canine ventricular myocytes (Bányász et al. 2003). A recurrent problem of the patch clamp-based electrophysiological studies is that kinetic properties of an ion channel can be accurately determined by applying rectangular voltage pulses, whereas the current profile displayed during an actual AP can be visualized only using AP voltage clamp conditions. A transitional approach can be the application of voltage ramps - as was the case in this study. Thus, we have measured human $I_{\mathrm{Ca}, \mathrm{L}}$ profiles under AP voltage clamp conditions, most kinetic properties of $I_{\mathrm{Ca}, \mathrm{L}}$ (voltage- and time-dependence of inactivation and recovery) using rectangular voltage pulses, and voltage-dependence of deactivation using both rectangular voltage pulses and voltage ramps. The $-9.6 \mathrm{mV}$ of midpoint potential obtained for voltage-dependence of deactivation using voltage ramps was close to that measured previously with rectangular pulses $(-12.7 \mathrm{mV})$. Although the distorting effect of inactivation could not be fully eliminated at the positive potential range in these experiments, the 0.042 minimum and the 0.84 maximum values estimated for the $I_{\mathrm{r}} / I_{\mathrm{P} 1}$ ratios indicate that under ramp conditions the majority of channels closed indeed via deactivation. This is further supported by the finding that the current grew back close to its initial value on the effect of the depolarizing ramp starting from $-40 \mathrm{mV}$ : the estimated maximum of the 
$I_{\mathrm{P} 2} / I_{\mathrm{P} 1}-V_{\mathrm{m}}$ curve was $0.88 \pm 0.03$. Thus, the development of the second current peak cannot be ascribed to recovery of the inactivated channel pool at the negative voltage range, since the time elapsed between the first and second $I_{\mathrm{Ca}, \mathrm{L}}$ peaks in the ramp protocol $(<20 \mathrm{~ms})$ was too short comparing to the $61.9 \mathrm{~ms}$ recovery time constant obtained for $I_{\mathrm{Ca}, \mathrm{L}}$. Of course, this argumentation is not valid for the positive membrane potential range where inactivation is the most important mechanism of closing the channels. This is the reason why the estimated minimum of the $I_{P 2} / I_{P 1}-V_{\mathrm{m}}$ curve in Figure $3 c$ and $f$ were $0.3 \pm 0.01$ and 0.34 , respectively. Therefore, the voltage-dependence of $I_{\mathrm{P} 2} / I_{\mathrm{P} 1}$ (shown in Fig. $3 \mathrm{c}$ and simulated in Fig. 3f) cannot be related to any discrete transition of the channel gating, rather it illustrates that a deeper incisura results in a higher amplitude of the second $I_{\mathrm{Ca}, \mathrm{L}}$ peak in EPI APs.

Although the relationship between the AP configuration and the underlying ionic currents is not fully understood, several reports were published on the impact of the AP configuration on $I_{\mathrm{Ca}, \mathrm{L}}$ profile. Early studies, based on traditional voltage clamp experiments using rectangular command pulses, concluded that an increased driving force caused by phase- 1 repolarization can maintain a sustained component of $I_{\mathrm{Ca}, \mathrm{L}}$ during the plateau. This prediction was justified experimentally under AP voltage clamp conditions in guinea pig (Arreola et al. 1991, Linz \& Meyer 1998) and rabbit (Yuan et al. 1996, Linz \& Meyer 2000) ventricular cells. Arreola et al. also reported a sustained component of $I_{\mathrm{Ca}, \mathrm{L}}$ during the plateau of the AP in guinea pig ventricular cells, but, in contrast to our results, reactivation of $I_{\mathrm{Ca}, \mathrm{L}}$ was not detected in that study (Arreola et al. 1991). They proposed that the increased driving force for calcium ions (resulting from partial repolarization during the incisura) and the voltagedependent recovery from inactivation of $I_{\mathrm{Ca}, \mathrm{L}}$ at plateau potentials are responsible for the sustained $I_{\mathrm{Ca}, \mathrm{L}}$. Similar results and conclusions were drawn by Yuan et al. (1996) in rabbit, and by Linz \& Meyer (2000) in guinea pig, rabbit, and rat myocytes. In contrast to these reports, the Luo-Rudy model predicts only a minor sustained component during canine ventricular AP (Luo \& Rudy 1994). Indeed, in our experiments performed in human cardiomyocytes, only negligible sustained component of $I_{\mathrm{Ca}, \mathrm{L}}$ was observed during the plateau. From this point of view our present results are in accordance with those of Bányász et al. (2003) and Zygmunt et al. (1997), who found no sustained component of $I_{\mathrm{Ca}, \mathrm{L}}$ in canine ventricular myocytes. This is not surprising taking into account the striking similarities in AP morphology observed between canine and human ventricular cells.

The question arises, if shifting the membrane potential toward more negative voltages does not increase $I_{\mathrm{Ca}, \mathrm{L}}$ during the plateau of the AP, what is the effect of phase1 repolarization on the profile of $I_{\mathrm{Ca}, \mathrm{L}}$ ? The first consequence of the early repolarization is the voltagedependent deactivation of L-type calcium channels. The fraction of channels driven into the deactivated state is primarily determined by the depth of the incisura, and probably by the rate of repolarization. Reopening of these channels after the incisura may contribute to development of the dome. Another fraction of calcium channels that may be involved in formation of the dome when reopening is that pool of channels which had already been inactivated before the incisura and thus may recover from inactivation during the incisura. The number of these channels depends on the depth as well as the duration of the incisura. According to our results a deeper and longer incisura may yield a larger population of calcium channels ready to reopen during the dome. Thus, ultimately, the large density of $I_{\text {to }}$, known to be present in human EPI myocytes, is responsible for the spike-and-dome configuration of the AP in these cells (Wettwer et al. 1994, Nabauer et al. 1996).

We have shown in this study that L-type calcium channels can, in fact, reopen during the AP plateau in human ventricular cells, which, in turn, may have secondary effects on AP configuration by controlling the activity of several voltage- and calcium-dependent ion currents. Indeed, reopening of calcium channels has been proposed to be involved in generation of early afterdepolarizations (January et al. 1988, January \& Riddle 1990, Wit \& Rosen 1992). Although we did not analyse afterdepolarizations under AP clamp conditions, our results are congruent with this hypothesis as early afterdepolarizations usually arise from membrane potentials more negative than the deepest point of the incisura.

Financial support for the studies was obtained from grants from the Hungarian Research Found (OTKA-T037332, OTKA-T037334, OTKA-T043182), Hungarian Ministry of Health (ETT-06031/2003) and from the National Research and Development Programs (NKFP-1A/0011/2002).

\section{References}

Arreola, J., Dirksen, R.T., Shieh, R.C., Williford, D.J. \& Sheu, S.S. 1991. $\mathrm{Ca}^{2+}$ current and $\mathrm{Ca}^{2+}$ transients under action potential clamp in guinea pig ventricular myocytes. Am J Physiol 261, C393-C397.

Bányász, T., Fülöp, L., Magyar, J., Szentandrássy, N., Varró, A. \& Nánási, P.P. 2003. Endocardial versus epicardial differences in L-type calcium current in canine ventricular myocytes studied by action potential voltage clamp. Cardiovasc Res 58, 66-75.

Brahmajothi, M.V., Campbell, D.L., Rasmusson, R.L. et al. 1999. Distinct transient outward current $\left(\mathrm{I}_{\mathrm{to}}\right)$ phenotypes and distribution of fast-inactivating potassium channel alpha 
subunit in ferret left ventricular myocytes. J Gen Physiol 113, 581-600.

Doerr, T., Denger, R., Doerr, A. \& Trautwein W. 1990. Ionic currents contributing to the action potential in single ventricular myocytes of the guinea pig studied with action potential clamp. Pflügers Arch 416, 230-237.

Furukawa, T., Kimura, S., Furukawa, N., Bassett, A.L. \& Myerburg, R.J. 1992. Potassium rectifier currents differ in myocytes of endocardial and epicardial origin. Circ Res 70, 91-103.

Hamill, O.P., Marty, A., Neher, E., Sakmann, B. \& Sigworth, F.J. 1981. Improved patch-clamp techniques for high resolution current recording from cells and cell-free membrane patches. Pflügers Arch 391, 85-100.

Ibarra, J., Morley, G.E. \& Delmar, M. 1991. Dynamics of the inward rectifier $\mathrm{K}^{+}$current during the action potential of guinea pig ventricular myocytes. Biophys J 60, 1534-1539.

January, C.T. \& Riddle, M.J. 1990. Early afterdepolarizations: mechanism of induction and block; a role for L-type $\mathrm{Ca}^{2+}$ current. Circ Res 64, 977-990.

January, C.T., Riddle, M.J. \& Salata, J.J. 1988. A model for early afterdepolarizations: induction with the $\mathrm{Ca}^{2+}$-channel agonist Bay K 8644. Circ Res 62, 563-571.

Kass, R.S. \& Sanguinetti, M.C. 1984. Inactivation of calcium channel current in the calf cardiac Purkinje fiber: evidence for voltage- and calcium-mediated mechanisms. J Gen Physiol 84, 705-726.

Krishnan, S.C. \& Antzelevitch, C. 1991. Sodium channel block produces opposite electrophysiological effects in canine ventricular epicardium and endocardium. Circ Res 69, 277291.

Linz, K.W. \& Meyer, R. 1998. Control of L-type calcium current during the action potential of guinea-pig ventricular myocytes. J Physiol 513, 425-442.

Linz, K.W. \& Meyer, R. 2000. Profile and kinetics of the L-type calcium current during the cardiac ventricular action potential compared in guinea-pigs, rats and rabbits. Pflügers Arch 439, 588-599.

Litovsky, S.H. \& Antzelevitch, C. 1988. Transient outward current prominent in canine ventricular epicardium but not endocardium. Circ Res 62, 116-126.

Litovsky, S.H. \& Antzelevitch, C. 1990. Differences in the electrophysiological response of canine ventricular subendocardium and subepicardium to acetylcholine and isoproterenol. Circ Res 67, 615-627.

Liu, D.W. \& Antzelevitch, C. 1995. Characteristics of the delayed rectifier current $\left(\mathrm{I}_{\mathrm{Kr}}\right.$ and $\left.\mathrm{I}_{\mathrm{Ks}}\right)$ in canine ventricular epicardial, midmyocardial and endocardial myocytes. A weaker $\mathrm{I}_{\mathrm{Ks}}$ contributes to the longer action potential of the M cell. Circ Res 76, 351-365.

Liu, D.W., Gintant, G.A. \& Antzelevitch, C. 1993. Ionic bases for electrophysiological distinctions among epicardial, midmyocardial, and endocardial myocytes from the free wall of the canine left ventricle. Circ Res 72, 671-687.

Luo, C.H. \& Rudy, L. 1994. A dynamic model of the cardiac ventricular action potential. Circ Res 74, 1071-1096.

Magyar, J., Iost, N., Körtvély, Á. et al. 2000. Effects of endothelin-1 on calcium and potassium currents in undiseased human ventricular myocytes. Pflügers Arch 441, 144-149.

Nabauer, M., Beuckelmann, D.J., Uberfuhr, P. \& Steinbeck, G. 1996. Regional differences in current density and ratedependent properties of the transient outward current in subepicardial and subendocardial myocytes of human left ventricle. Circulation 93, 168-177.

Stankovicova, T., Szilard, M., Scheerder, I.D. \& Sipido, K.R. 2000. M cells and transmural heterogeneity of action potential configuration in myocytes from the left ventricular wall of the pig heart. Cardiovasc Res 45, 952-960.

Volk, T., Nguyen, T.H.D., Schultz, J.H. \& Ehmke, H. 1999. Relationship between transient outward $\mathrm{K}^{+}$current and $\mathrm{Ca}^{2+}$ influx in rat cardiac myocytes of endo- and epicardial origin. J Physiol 519, 841-850.

Wettwer, E., Amos, G.J., Posival, H. \& Ravens, U. 1994. Transient outward current in human ventricular myocytes of subepicardial and subendocardial origin. Circ Res 75, 473482.

Wit, A.L. \& Rosen, M.R. 1992. Afterdepolarizations and triggered activity: distinction from automaticity as an arrhythmogen mechanism. In: H.A. Fozzard, E. Haber, R.B. Jennings, A.M. Katz \& H.E. Morgan (eds) The Heart and Cardiovascular System, pp. 2148-2149. Raven Press, New York.

Yuan, W., Ginsburg, K.S. \& Bers, D.M. 1996. Comparison of sarcolemmal calcium channel current in rabbit and rat ventricular myocytes. J Physiol 493, 733-746.

Zaza, A., Rocchetti, M., Brioschi, A., Cantadori, A. \& Ferroni, A. 1998. Dynamic $\mathrm{Ca}^{2+}$-induced inward rectification of $\mathrm{K}^{+}$ current during the ventricular action potential. Circ Res 82, 947-956.

Zygmunt, A.C., Robitelle, D.C. \& Eddlestone, G.T. 1997. I $_{\text {to1 }}$ dictates behavior of $\mathrm{I}_{\mathrm{Cl}(\mathrm{Ca})}$ during early repolarization of canine ventricle. Am J Physiol 273, H1096-H1106. 